\title{
Quantum scissors: teleportation of single-mode optical states by means of a nonlocal single photon
}

\author{
S. A. Babichev, J. Ries, A. I. Lvovsky \\ Fachbereich Physik, Universität Konstanz, D-78457 Konstanz, Germany
}

(October 22, 2018)

\begin{abstract}
We employ the quantum state of a single photon entangled with the vacuum $\left(|1\rangle_{A}|0\rangle_{B}-|0\rangle_{A}|1\rangle_{B}\right)$, generated by a photon incident upon a symmetric beam splitter, to teleport single-mode quantum states of light by means of the Bennett protocol. Teleportation of coherent states results in truncation of their Fock expansion to the first two terms. We analyze the teleported ensembles by means of homodyne tomography and obtain fidelities of up to 99 per cent for low source state amplitudes. This work is an experimental realization of the quantum scissors device proposed by Pegg, Phillips and Barnett (Phys. Rev. Lett. 81, 1604 (1998))

PACS numbers: 03.67.Hk, 03.65.Ud, 03.65.Wj, 42.50.Dv
\end{abstract}

a. Introduction Quantum teleportation (QT) is the transport of an unknown quantum state $|\phi\rangle$ over arbitrary distances by means of dual classical and EinsteinPodolsky-Rosen (EPR) channels. To perform teleportation, the sender, Alice, and the receiver, Bob, prearrange the sharing of an EPR-correlated pair of particles. Alice makes a joint measurement on her EPR particle and the source state and sends Bob the classical result of this measurement. Knowing this, Bob can convert the state of his EPR particle into an exact copy of the source state. In this way neither Alice nor Bob obtain any information about the state $|\phi\rangle$ but this state is available at Bob's location for future use.

Although teleportation of macroscopic objects is far beyond modern technology, quantum teleportation of microscopic states may find its application in the observable future as a key ingredient of quantum communication and computation. It can be used in combination with non-deterministic computational gates to enhance their success probability, thus making schemes involving such gates scalable and efficient [1]. This is the role QT plays in the recently proposed scheme for efficient quantum computation with linear optics [2].

After its proposal in 1993 by Bennett et al. [3], QT has been implemented experimentally on discrete- [4,5] and continuous-variable optical states [6] as well as on molecular spins [7]. All these schemes used an EPR pair maximally entangled in a Hilbert space which is isomorphic to the Hilbert space of the source state. This identity allows, in principle, exact replication of the source state by Bob.

An interesting extension of the Bennett protocol arises, however, if the source state lives in the Hilbert space of higher dimension than the EPR pair. In this case all terms of the source state associated with the dimensions beyond that of the EPR pair will be "cut off" from the teleported state. This is known as the quantum scissors (QS) effect first described theoretically in 1998 by Pegg, Phillips, and Barnett [8] and implemented experimen- tally in the present work.

In the heart of our teleportation experiment there is an EPR pair implemented by a nonlocal single photon state $\left|\Psi^{-}\right\rangle=\frac{1}{\sqrt{2}}(|1\rangle|0\rangle-|0\rangle|1\rangle)$ which is generated when a single photon $|1\rangle$, incident upon a symmetric beam splitter, entangles itself with the vacuum $|0\rangle$. It is remarkable that our EPR ensemble is formed by just one particle; yet this is a maximally entangled state in the two-dimensional Hilbert space defined by basis vectors $|0\rangle$ and $|1\rangle$.

We apply the state $\left|\Psi^{-}\right\rangle$to teleport arbitrary singlemode quantum states of the electromagnetic field that belong to the Hilbert space of infinite dimension. If a random quantum state is given by $|\phi\rangle=\sum a_{n}|n\rangle$ in the number (Fock) state basis, the scissors effect will truncate the above series, leading to the output of a form $\left|\phi_{\text {out }}\right\rangle=$ $a_{0}|0\rangle+a_{1}|1\rangle$. In simpler words, the higher number terms cannot reach Bob because there is never more than one photon in the original EPR state $\left|\Psi^{-}\right\rangle$.

In our actual experiment, the role of the source ensemble was played by a coherent state $|\alpha\rangle$. Since this state has an infinite number of terms in its Fock expansion, it is well suitable for demonstrating quantum scissors; on the other hand it is readily available from the source laser. Alice's Bell-state measurement is performed by overlapping the source state and her share of the EPR state on a beam splitter and measuring the number of photons in each output (Fig. 1). Events in which the detector $D_{1}$ registers exactly one photon while $D_{2}$ registers zero photons correspond to the two-mode state $\left|\Psi^{-}\right\rangle_{12}$ entering Alice's apparatus in modes 1 and 2. If this is the case, Bob's share of the EPR state (mode 3 ) is in the state $\left|\phi_{\text {out }}\right\rangle$ so no additional manipulations are required from Bob to complete the QT protocol. Restricting to these events, we perform a homodyne measurement on the teleported ensembles in order to characterize them and determine the teleportation fidelity.

Full implementation of the scissors protocol requires, in particular, highly efficient single-photon detectors capable of determining the number of incident photons. Al- 
though such detectors are currently being developed [9], they are not widely available. Fortunately, the protocol exhibits surprisingly good fidelity even with regular, non-discriminating single-photon detectors as long as the amplitude of the source state is sufficiently small [10,11.

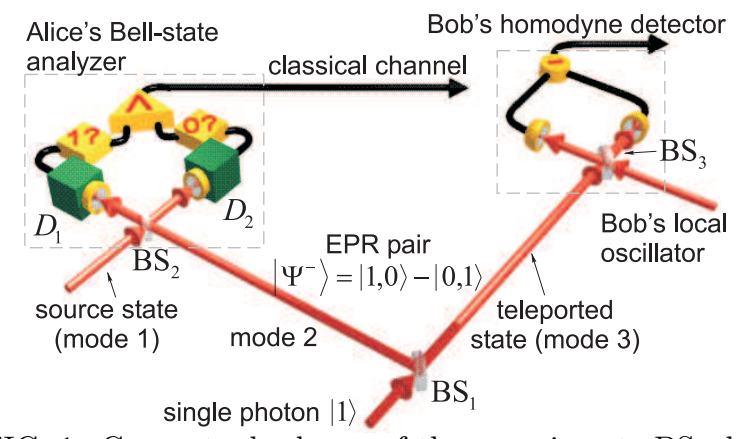

FIG. 1. Conceptual scheme of the experiment. $\mathrm{BS}_{i}$, beam splitters; $D_{i}$, single photon detectors.

The QS protocol finds its direct application as an integral part of the single-rail version of the linear optical quantum computer recently proposed by Lund and Ralph [15. Apart from the quantum gate efficiency enhancement discussed earlier, this technique is useful for preparing quantum bits of arbitrary value. Although conditional preparation of superpositions $a_{0}|0\rangle+a_{1}|1\rangle$ has been reported earlier [12 13], the present scheme is highly efficient, employs only passive optics and can be extended to multi-photon ensembles 14.

In a related experiment, Lombardi et al. recently used the nonlocal photon for partial teleportation of a singlephoton qubit encoded in a dual optical channel. One part of the entangled source state was teleported to Bob by means of a Bell measurement while the other was transported to Bob directly [16]. The main difference between Ref. [16] and this work is that the former required a direct quantum channel between Alice and Bob to complete the transfer of a quantum state, whereas the latter is a straightforward implementation of the Bennett protocol [3] in the form of quantum scissors [8].

We note that our teleportation scheme is of a priori nature in the context of the discussion [17], i.e. teleportation events need not be postselected according to the result of Bob's measurements. Whenever Alice obtains a positive result of the Bell measurement, it is known that the teleportation has been successful and the teleported state is available for future use.

b. Theory The practical implementation of the QS protocol requires a source of single photons in a pure optical mode matching that of the source ensemble. We solve this task by means of conditional measurement on a down-converted pair: the single photon state $|1\rangle$ is prepared in the signal channel of the down-converter when its counterpart is detected in the idler [18,19]. Theoretical treatment of the scissors protocol in this configuration has been elaborated by Özdemir et al. [11,20, so here we only present a brief overview.

Preparation of the single photon state is imperfect: dark counts of our trigger detector $\left(D_{T}\right)$ and optical losses result in a statistical mixture of one photon and no photon instead of a pure $|1\rangle$ state. The ensemble entering the first beam splitter is therefore

$$
\hat{\rho}_{|1\rangle}=\eta_{|1\rangle}|1\rangle\left\langle 1\left|+\left(1-\eta_{|1\rangle}\right)\right| 0\right\rangle\langle 0|,
$$

$\eta_{|1\rangle}$ being the preparation efficiency.

The density matrix of the EPR pair used for teleportation $\hat{\rho}^{\text {epr }}$ can be found by applying the beam splitter transformation operator

$$
\begin{aligned}
\hat{B}|m, n\rangle & =\sum_{j, k=0}^{m, n} \sqrt{\frac{(j+k) !(m+n-j-k) !}{m ! n !}}\left(\begin{array}{c}
m \\
j
\end{array}\right)\left(\begin{array}{c}
n \\
k
\end{array}\right) \\
& \times(-1)^{k} 2^{-(n+m) / 2}|j+k, m+n-j-k\rangle
\end{aligned}
$$

to the incident combination of $\hat{\rho}_{|1\rangle}$ and the vacuum:

$$
\hat{\rho}^{\text {epr }}=\hat{B}\left(|0\rangle\langle 0| \otimes \hat{\rho}_{|1\rangle}\right) \hat{B}^{\dagger}
$$

The source coherent state and one of the EPR "particles" enter Alice's apparatus where they undergo further transformation via another beamsplitter. After this transformation, the density matrix of the 3-mode ensemble can be written as

$$
\hat{\rho}_{123}=\hat{B}_{12}\left(|\alpha\rangle_{1}\left\langle\left.\alpha\right|_{1} \otimes \hat{\rho}_{23}^{\mathrm{epr}}\right) \hat{B}_{12}^{\dagger},\right.
$$

where the subscripts refer to the optical modes according to Fig. 1.

The first two modes of $\hat{\rho}_{123}$ are subjected to measurements via single-photon detectors. A non-discriminating detector of quantum efficiency $\eta_{\mathrm{SPD}}$ is described by the following positive operator-valued measure (POVM):

$$
\begin{aligned}
\hat{\Pi}^{\text {no-click }} & =\sum_{n=0}^{\infty}\left(1-\eta_{\mathrm{SPD}}\right)^{n}|n\rangle\langle n| \\
\hat{\Pi}^{\text {click }} & =\hat{1}-\hat{\Pi}^{\text {no-click }} .
\end{aligned}
$$

This measurement leads to a collapse of $\hat{\rho}_{123}$ projecting it in the event of a "click" in detector $D_{1}$ and "no click" in detector $D_{2}$ upon the following non-normalized ensemble in Bob's channel:

$$
\hat{\rho}_{\text {out }}=\operatorname{Tr}_{12}\left(\hat{\rho}_{123} \hat{\Pi}_{1}^{\text {click }} \hat{\Pi}_{2}^{\text {no-click }}\right) .
$$

The probability of a teleportation event is given by $p_{\text {tel }}=$ $\operatorname{Tr}\left(\hat{\rho}_{\text {out }}\right)$.

Imperfect spatial, spectral or temporal mode matching between Alice's share of the nonlocal single photon and the source state $|\alpha\rangle$ leads to partial distinguishability and more classical-like behavior, reducing the teleportation fidelity. In case of a complete mode mismatch, the 
behavior of the system is fully described by a semiclassical model in which photons act like particles with no wave properties. Each beam splitter distributes the incident photons randomly into the output channels. The correlated photon number distribution in the three modes can be calculated according to the laws of classical statistics. From this distribution we infer the probability $p_{\text {tel }}^{\text {sc }}$ of the positive Bell measurement outcome as well as the conditional probability $p_{\text {out }}^{\text {sc }}$ that Bob's mode contains a photon. The ensemble received by Bob can then be expressed as a density matrix

$$
\hat{\rho}_{\text {out }}^{\mathrm{sc}}=\left(\begin{array}{cc}
1-p_{\text {out }}^{\mathrm{sc}} & 0 \\
0 & p_{\text {out }}^{\mathrm{sc}}
\end{array}\right) .
$$

In the actual case of partial mode matching, the output ensemble is a mixture of those calculated via classical and semiclassical models

$$
\hat{\rho}^{\mathrm{Bob}}=M p_{\text {out }} N\left[\hat{\rho}_{\text {out }}\right]+(1-M) p_{\text {out }}^{\mathrm{sc}} N\left[\hat{\rho}_{\text {out }}^{\mathrm{sc}}\right],
$$

where $M$ is the mode matching factor [19] and $N[\hat{\rho}]$ denotes normalization.

Imperfections in the homodyne detection of the teleported state such as poor mode matching between the local oscillator and the signal, linear losses, inefficient photodiodes or imperfect balance can all be modeled by a single beam splitter with one empty input in the signal beam with a reflectivity $\eta_{\mathrm{HD}}$ (generalized Bernoulli transformation) [19,21].

c. Experimental apparatus The setup for preparing the single-photon Fock state was the same as in our previous experiments 12 18]. A $82-\mathrm{MHz}$ repetition rate train of 1.6-ps pulses generated by a Spectra-Physics Ti:Sapphire laser at $790 \mathrm{~nm}$ was frequency doubled and directed into a beta-barium borate crystal for downconversion. The latter occurred in a type-one frequencydegenerate, but spatially non-degenerate configuration. The single-photon detector $D_{T}$, placed into the idler channel of the down-converter, detected photon-pair creation events and triggered all further measurements.

Pulses containing conditionally-prepared photons entered the optical arrangement shown in Fig. 1, which had to be maintained interferometrically stable throughout the experimental run. The coherent source state $|\alpha\rangle$ and the local oscillator for homodyne detection were provided by the master Ti:Sapphire laser. These two modes had to be matched, spatially and temporally, to the respective modes of the EPR pair. To this end, we modeled the single photon by a classical wave as described in 18, 19. The mode matching was then optimized by maximizing the visibility of the interference fringes observed in the beam splitter outputs. The visibility value provided a basis for a ballpark estimation of the mode matching factor $M$.

Further knowledge of the experimental parameters was gained through an auxiliary tomography measurement in which the ensemble arriving to Bob was characterized without conditioning on Alice's results. This ensemble is a statistical mixture of states $|0\rangle$ and $|1\rangle$ with the singlephoton fraction equal to $\eta_{|1\rangle} \eta_{\mathrm{HD}} / 2$. We found $\eta_{|1\rangle} \eta_{\mathrm{HD}} \approx$ 0.49

All single-photon detectors used were from PerkinElmer, SPCM-AQ series, with quantum efficiencies (including the filtering optics) of about $\eta_{\mathrm{SPD}} \approx 0.5$. The homodyne measurement of the teleported state was conditioned upon detectors $D_{1}$ and $D_{T}$ firing and the detector $D_{2}$ not firing. The digital logic employed featured rigorous synchronization control of the photon count events with respect to each other and to the master laser pulses. This helped us reduce the dark count contribution to a negligible level.

The time-domain homodyne detector used for characterizing the teleported state was described in 22]. For each value of $\alpha$ approximately 20000 events were collected. The phase of the local oscillator was varied with a piezoelectric transducer. The acquired data was used to calculate the density matrix $\hat{\rho}_{\text {out }}^{\exp }$ of the teleported ensemble by means of the quantum state sampling method [21. The teleportation fidelity was then evaluated as

$$
F=\left\langle\alpha\left|\hat{\rho}_{\text {out }}^{\exp }\right| \alpha\right\rangle .
$$

d. Results and discussion For conceptual verification of the teleportation protocol we performed a measurement run in which we varied the phase of the source state instead of the local oscillator. From the classical point of view, this action should not affect the optical field observed by Bob and therefore its quadrature statistics should remain constant. Yet we observed the optical phase of the teleported ensemble vary in accordance with that of the source (Fig. 2). This result is readily explained by quantum mechanics: by changing the source state phase Alice changes the conditions of the measurement performed on one of the members of the EPR pair. This has a nonlocal effect on the other member which is observed by Bob in his homodyne measurement.

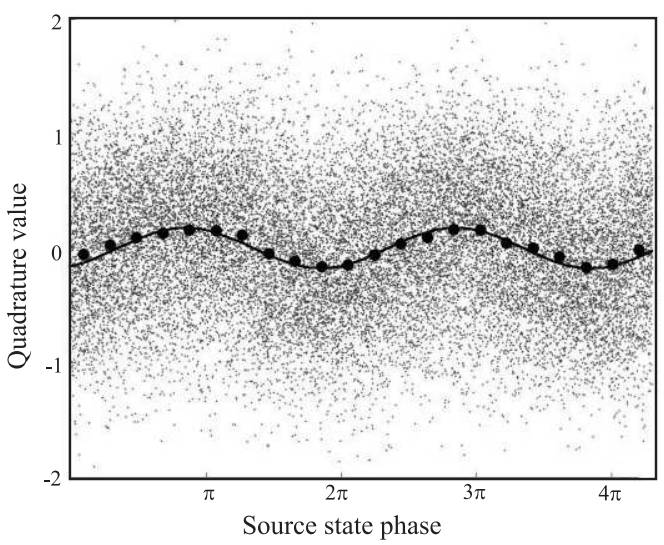

FIG. 2. Quadrature noise distribution of the teleported state measured with a balanced homodyne detector as a function of the source state phase. 
Fig. 3 shows the teleportation fidelity determined experimentally along with the theoretical fit calculated according to Eqs. (6)-(9). There were three fitting parameters: quantum efficiencies $\eta_{|1\rangle}$ and $\eta_{\mathrm{HD}}$ of the single photon preparation and the homodyne detection, respectively, and the mode matching factor $M$. By fitting these parameters with fixed $\eta_{\mathrm{HD}} \cdot \eta_{|1\rangle}$ we found $\eta_{\mathrm{HD}}=0.54$, $\eta_{|1\rangle}=0.9, M=0.56$. Note that the value of $\eta_{\mathrm{HD}}$ includes not only the homodyne detector efficiency per se, but also the mode matching of Bob's ensemble with the local oscillator.

Along with the data pertinent to the actual experiment, Fig. 3 also shows the behavior of the fidelity factor for the idealized quantum-mechanical model with number discriminating detectors and the semiclassical particle model discussed above. All three models exhibit similar qualitative behavior. If the source state is vacuum, a photon detected by Alice must originate from the EPR pair, so Bob receives no photons. The ensemble arriving at Bob's station is in the vacuum state, and the teleportation fidelity is perfect. For high $\alpha$, the input state has almost vanishing vacuum and single-photon terms, the only components of the truncated teleported ensemble. The teleported ensemble is then practically orthogonal to the source state, and the fidelity is low.

Our experimentally measured fidelity is always higher than that predicted semiclassically, showing the importance of quantum nonlocal effects. A remarkable feature is that for low values of $\alpha$ the value of $F$ is very high, up to 99 per cent. To our knowledge, this is the highest fidelity ever achieved in experimental QT.

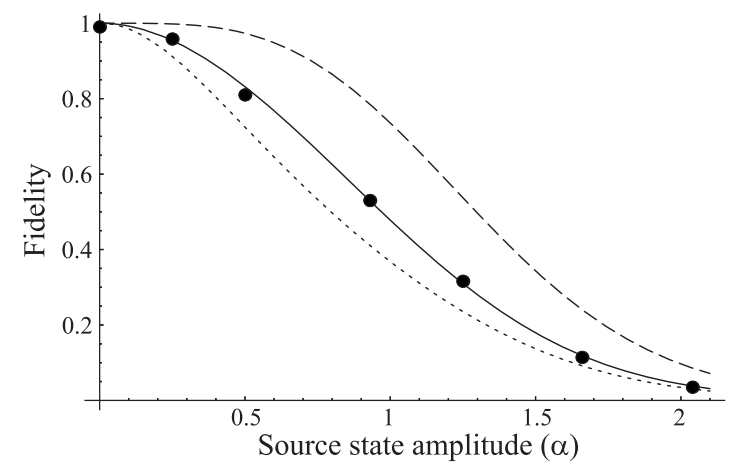

FIG. 3. Teleportation fidelity as a function of the amplitude $\alpha$ of the coherent source state. Shown are the experimental data and the theoretical fit (solid line), the idealized quantum mechanical model with number discriminating detectors (dashed line) and the semiclassical particle model (dotted line).

e. Conclusion We reported an experimental realization of quantum scissors, i.e. teleportation of single-mode optical ensembles using the nonlocal single photon state as the EPR pair. The teleported state was examined by homodyne measurement and the fidelity was found to be well above the classical limit. Since we did not postselect the teleportation events according to Bob's results, this experiment is of a priori nature. To our knowledge, this is the first QT experiment in which the Bell measurement was done in a discrete, and the characterization of the teleported state in a continuous basis.

In perspective we plan to improve our teleportation fidelity by using number discriminating photon detectors [9]. Another possibility would be to extend the QS protocol to synthesize arbitrary truncated superpositions of Fock states $a_{0}|0\rangle+\ldots+a_{n}|n\rangle$ 14,23. The nonlocal single photon $|0,1\rangle+|1,0\rangle$ is worth further investigation from the point of view of quantum nonlocality 24.

This work was sponsored by the Deutsche Forschungsgemeinschaft and the Optik-Zentrum Konstanz.

* Email: Alex.Lvovsky@uni-konstanz.de

[1] D. Gottesman, I. L. Chuang, Nature 402, 390(1999)

[2] E. Knill, R. Laflamme, G. J. Milburn, Nature (London) 409, 46(2001)

[3] C. H. Bennett et al. Phys. Rev. Lett. 70, 1895 (1993).

[4] D. Bouwmeester et al., Nature (London) 390, 575(1997)

[5] D. Boschi et al., Phys. Rev. Lett. 80, 1121 (1998)

[6] A. Furusawa et al., Science 282, 706 (1998).

[7] M. A. Nielsen, E. Knill, and R. Laflamme, Nature (London) 396, 52 (1998)

[8] D. T. Pegg, L. S. Phillips, and S. M. Barnett, Phys. Rev. Lett. 81, 1604 (1998)

[9] S. Takeuchi et al., Appl. Phys. Lett 74, 1063 (1999)

[10] S. M. Barnett and D. T. Pegg, Phys. Rev. A 60, 4965 (1999)

[11] S. K. Özdemir et al., Phys. Rev. A 64, 063818 (2001)

[12] A. I. Lvovsky , J. Mlynek, Phys. Rev. Lett. 88, 250401 (2002).

[13] K. J. Resch, J. S. Lundeen, and A. M. Steinberg, Phys. Rev. Lett. 88, 113601 (2002)

[14] M. Koniorczyk et al., Phys. Rev. A 62, 013802 (2001); C. J. Villas-Boas et al., Phys. Rev. A 63, 055801 (2001)

[15] A. P. Lund, T. C. Ralph, quant-ph/0205044

[16] E. Lombardi et al., Phys. Rev. Lett. 88, 070402 (2002)

[17] S. L. Braunstein, H. J. Kimble, Nature (London) 394, 841 (1998)

[18] A. I. Lvovsky et al. Phys. Rev. Lett. 87, 050402 (2001)

[19] T. Aichele, A. I. Lvovsky and S. Schiller, Eur. Phys. J. D 18, 237 (2002).

[20] S. K. Özdemir et al., quant-ph/0206072

[21] U. Leonhardt, Measuring the quantum state of light, Cambridge University Press, 1997.

[22] H. Hansen et al., Opt. Lett. 26, 1714 (2001).

[23] M. Dakna et al., Phys. Rev. A 59, 1658 (1999);

[24] S. M. Tan, D. F. Walls, and M. J. Collett, Phys. Rev. Lett. 66, 252 (1991); L. Hardy, Phys. Rev. Lett. 73, 2279 (1994); K. Jacobs and P. L. Knight, Phys. Rev. A 54, 3738 (1996) 